\title{
DECOMPOSIÇÃO DA ELASTICIDADE-PREÇO NO VAREJO COM USO DE DADOS ESCANEADOS
}

\author{
Delane Botelho \\ Escola Brasileira de Administração Pública e de \\ Empresas (EBAPE/FGV) \\ Rio de Janeiro - RJ \\ dbotelho@,fgv.br
}

Recebido em 07/2003; aceito em 04/2005 após 1 revisão Received July 2003; accepted April 2005 after one revision

\begin{abstract}
Resumo
O objetivo deste artigo é estimar a elasticidade-preço da demanda decomposta em elasticidade-preço da escolha da marca e da quantidade comprada, e discutir as implicações desta decomposição para uma categoria específica de bem de consumo. Para isto foram utilizados dados escaneados de uma amostra de domicílios no contexto varejista brasileiro. Dois modelos foram usados: o modelo logit condicional baseado na maximização da utilidade do domicílio para a decisão de escolha da marca e o modelo clássico de regressão linear para a decisão da quantidade a comprar. No que diz respeito à validação, o primeiro modelo demonstrou uma satisfatória capacidade de previsão da participação de mercado das marcas. Implicações gerenciais incluem específicas decisões e ações de preço para as marcas, já que a natureza da decomposição das elasticidades-preço varia entre marcas.
\end{abstract}

Palavras-chave: elasticidade-preço; modelo logit; dados escaneados.

\begin{abstract}
This article focuses on the estimation of the total price-elasticity of demand, decomposed in brand choice and quantity price-elasticity, and it discusses the implications of such decomposition for a specific product category, using a scanner data sample of Brazilian households. Two models were used: the brand choice decision was modeled through the conditional logit based on household utility maximization; the decision of how much to buy was modeled through classical linear regressions. Regarding the validity, the first model demonstrated a satisfactory prevision power of brand market share. Managerial implications include specific price decisions to each brand, as the nature of the priceelasticity decomposition varies among brands.
\end{abstract}

Keywords: price-elasticity; logit model; scanner data. 


\section{Introdução}

A variação de preços pode ter impacto substancial no comportamento de curto prazo de compra do consumidor para bens de consumo. No varejo, especialmente em supermercados, decisões de promoções de preço podem ser de alta complexidade para os gerentes de categoria, aqueles responsáveis pelo marketing das marcas de determinada categoria de produto. Assim, a sensibilidade do consumidor ao preço é uma medida essencial na otimização de decisões de apreçamento das empresas. Esta sensibilidade é medida tradicionalmente pela elasticidade-preço total da demanda ( $\left.E t_{p p}\right)$, que representa a variação da quantidade demandada, dada a variação do preço, num ponto específico da curva de demanda:

$$
E t_{p p}=\frac{\partial \mathrm{Q} / \mathrm{Q}}{\partial \mathrm{P} / \mathrm{P}}=\frac{\partial \mathrm{Q} / \partial \mathrm{P}}{\mathrm{Q} / \mathrm{P}}
$$

em que $\partial \mathrm{Q} / \partial \mathrm{P}$ exprime a variação da quantidade comprada resultante da variação unitária do preço.

No entanto, esta abordagem tradicional de estimação da elasticidade-preço apresenta uma limitação: não soluciona as carências de informação dos gerentes de marketing no varejo, já que eles sabem que a decisão de compra de uma marca não se dá apenas na dimensão da quantidade a ser comprada, mas também na escolha da marca a comprar (Krishnamurthi \& Raj, 1988). Há uma resposta do consumidor ao preço em cada um destes dois estágios do comportamento de compra e a natureza destas respostas depende do papel que o nível de preços assume, ou seja, depende da sensibilidade do consumidor ao preço em cada estágio (escolha da marca e quantidade comprada).

Outra limitação presente em muitos estudos de elasticidade-preço é a agregação da função de demanda para um setor ou para uma empresa, desconsiderando informações desagregadas, no nível individual de comprador. Um forte motivo desta limitação era a indisponibilidade de dados desagregados no Brasil até a década de 1990. Esta limitação pode ser superada atualmente por dados escaneados de itens de marcas específicas (Gijsbrechts, 1993). Tais dados são obtidos quando o produto é passado por um scanner a laser, que procede a leitura ótica do código de barras da embalagem e/ou do cartão de fidelidade do cliente no checkout da loja. Esta desagregação no nível de domicílio possibilita maior variabilidade, o que é uma vantagem em análises estatísticas, já que a agregação de dados reduz a variância das variáveis aleatórias e tende a mascarar a relação entre preço e vendas (Kim, 1996).

Este artigo pretende superar essas duas limitações da literatura de otimização de promoções no Brasil, abordando a escolha do consumidor pela marca e quantidade comprada, tendo o preço como principal variável mercadológica influenciadora destas respostas. Como tais escolhas afetam as elasticidades e o preço representa a principal variável deste artigo, o trabalho empírico foca a estimação conjunta da elasticidade-preço da escolha da marca e elasticidade-preço da quantidade comprada utilizando-se dados escaneados de uma rede varejista brasileira.

Os subscritos usados aqui são os seguintes: cada domicílio $(i)$ possui ocasiões de compra $(t)$ na categoria num período total de $\mathrm{T}$ semanas. Supondo-se um corte transversal com $\mathrm{N}$ ocasiões de compra (it), o domicílio $i$ escolherá a alternativa (marca) $p$ que maximizará sua utilidade, dentro de um conjunto de $\mathrm{P}$ alternativas. Os termos alternativa e marca são usados indistintamente. 
Além de $E t_{p p}$, que representa a elasticidade-preço total da demanda, as seguintes notações serão aqui usadas: $E q_{p p}=$ elasticidade-preço da quantidade comprada, $E q_{p j}=$ elasticidadepreço cruzada da quantidade comprada, $E e_{p p}=$ elasticidade-preço da escolha da marca, $E e_{p j}=$ elasticidade-preço cruzada da escolha da marca.

\section{O Modelo}

No processo de compra, o consumidor se defronta com diversos tipos de decisão: a loja, a categoria do produto, o tipo de produto (como o sabor ou o modelo), a marca, a quantidade a comprar e quando comprar. A maioria destas decisões é estudada com modelos probabilísticos de escolha, como logit, probit, modelos generalizados de valor extremo e, mais recentemente, os modelos logit mistos com coeficientes aleatórios e procedimentos bayesianos (Chintagunta et al., 2001).

Neste artigo enfocam-se duas decisões relacionadas entre si: qual marca optar e quanto comprar. A escolha da marca pode depender dos preços e dos atributos das marcas disponíveis na loja, dentro da categoria estudada. A quantidade a ser comprada pode depender das características do domicílio, como a necessidade de consumo e a restrição orçamentária.

\subsection{Funções de utilidade e o modelo logit condicional}

A atratividade de uma marca é avaliada pelos domicílios em termos de um vetor de valores de atributos. Eles podem avaliar diferentes atributos para as marcas e atribuir diversos valores para o mesmo atributo da mesma alternativa. Segundo Ben-Akiva \& Lerman (1985, p.35), uma regra de decisão usada em modelos de escolha é a utilidade, definida como um índice de atratividade, ou seja, uma medida que o indivíduo tenta maximizar por meio de sua escolha. O conceito de utilidade pressupõe que os atributos sejam mensuráveis, definindo uma função objetiva que expresse a atratividade de uma alternativa pelos seus atributos. Também pressupõe que o consumidor apresente comportamento racional de compra.

Em vez de se derivar funções de demanda, trabalha-se diretamente com as funções de utilidade. Por exemplo, no problema de escolha de marcas de um domicílio com $\mathrm{P}$ alternativas, define-se uma função utilidade em termos de atributos das alternativas e características do domicílio do seguinte modo: $\mathrm{U}_{p}=\mathrm{U}\left(\mathrm{x}_{p}, \mathrm{w}_{i}\right)$, em que $\mathrm{U}_{P}$ refere-se à função utilidade de uma marca específica $p . \mathrm{x}_{p} \mathrm{e} \mathrm{w}_{i}$ referem-se ao atributo da marca $p$ e à característica do domicílio $i$, respectivamente.

$\mathrm{U}(\cdot)$ é uma função ordinal, isto é, a alternativa 1 , por exemplo, será escolhida se, e somente se, $\mathrm{U}_{1}>\mathrm{U}_{2} ; \mathrm{U}_{1}>\mathrm{U}_{3} ; \ldots ; \mathrm{U}_{1}>\mathrm{U}_{p}$. Assumindo-se uma função linear, tem-se, por exemplo, $\mathrm{U}_{p}=-\beta_{1} \mathrm{x}_{p}+\beta_{2} \mathrm{~W}_{i}$, em que $\beta_{1}$ e $\beta_{2}$ são parâmetros que expressam a preferência do consumidor. $\mathrm{Na}$ abordagem da utilidade, assume-se sempre que o consumidor escolhe a alternativa de mais elevada utilidade.

As utilidades são conceitos latentes (não observados pelo pesquisador) e apresentam componentes aleatório e determinístico. O primeiro é representado pelo termo de erro do modelo $\varepsilon$ e o determinístico é representado por, por exemplo, $V$. Sendo $\mathbf{z}_{i p}=\left[\begin{array}{lll}\mathbf{w}_{i} & \mathbf{x}_{p}\end{array}\right]$ o 
conjunto de vetores de atributos da marca e características do domicílio, $V_{i p}=\boldsymbol{\beta}^{\prime} \mathbf{z}_{i p}$ e $\mathrm{U}_{i p}=\mathrm{V}_{i p}+\varepsilon_{i p}$. No caso deste artigo, o domicílio se depara com mais de duas alternativas e há os dois vetores de variáveis $\left(\mathbf{w}_{i} \mathbf{x}_{p}\right.$ ). Assim, segundo Liao (1994, p.59), o modelo logit condicional é o mais apropriado, e se $\varepsilon_{i p}$ são assumidos (por conveniência analítica) como independentes e identicamente distribuídos (i.i.d.) pela distribuição de Gumbel, então a função densidade de probabilidade $F(\varepsilon)=\exp \left(-e^{-\varepsilon}\right)$ e:

$$
\mathrm{P}\left(Y_{i}=j\right)=\frac{e^{\boldsymbol{\beta}^{\prime} \mathbf{z}_{i j}}}{\sum_{p=1}^{P} e^{\boldsymbol{\beta}^{\prime} \mathbf{z}_{i p}}}
$$

refere-se ao modelo logit condicional (Liao, 1994, p.59), cujos parâmetros $\beta$ são calculados pelo método da máxima verossimilhança. Os termos que não variam entre as alternativas, ou seja, aqueles específicos aos indivíduos, são eliminados da probabilidade no modelo logit condicional. Então, precisa-se modificar tal modelo para que ele permita efeitos específicos aos indivíduos, por meio da criação de um conjunto de variáveis categóricas para as alternativas e multiplicação de cada uma delas por $\mathbf{w}_{i}$, que é comum a todas as alternativas, como no caso de uma característica domiciliar. O que se faz, portanto, é permitir que os coeficientes variem para cada alternativa, em vez de variarem apenas para as características individuais.

\subsection{Elasticidade-preço no modelo de escolha da marca}

A elasticidade agregada no modelo logit representa o efeito na probabilidade de escolha de um grupo de indivíduos em diferentes ocasiões de compra, devido à variação de um atributo qualquer (Ben-Akiva \& Lerman, 1985, p.111). Por exemplo, suponha-se que se queira saber o efeito de uma mudança na variável independente "preço" sobre a probabilidade de escolha da marca $p$. Para isto, define-se $\bar{P}(p)$ como a participação esperada do grupo de indivíduos que escolhe a alternativa $p$ nas várias ocasiões de compra:

$$
\bar{P}(p)=\frac{\sum_{i=1}^{N} P_{i}(p)}{N}
$$

em que $\mathrm{N}$ é número de observações (ocasiões de compra).

Suponha-se que se altere o valor de alguma variável $z_{i p k}$ para cada domicílio em cada ocasião de compra, de forma que $\partial z_{i p k} / z_{i p k}=\partial z_{i^{\prime} p k} / z_{i^{\prime} p k}=\partial z_{p k} / z_{p k}$, para todo $i, i^{\prime}=1,2, \ldots, \mathrm{N}$, onde $z_{p k}=\frac{1}{N} \sum_{i=1}^{N} z_{i p k}$. Isto corresponde a uma variação percentual uniforme em $z_{i p k}$ em todos os membros do grupo e em todas as ocasiões de compra. Para este tipo de variação a elasticidade será:

$$
E_{z_{p k}}^{\bar{P}_{i}(p)}=\frac{\sum_{i=1}^{N} P_{i}(p) E_{z_{i p k}}^{P_{i}(p)}}{\sum_{i=1}^{N} P_{i}(p)}
$$


que é simplesmente uma média ponderada das elasticidades individuais usando-se a probabilidade de escolha como pesos. Uma propriedade do modelo logit é que ele possui elasticidades cruzadas uniformes, isto é, as elasticidades de todas as alternativas, com respeito à variação num atributo que afeta apenas a utilidade da alternativa $p$, são iguais para todas as alternativas diferentes de $p$. Esta característica decorre da Independência de Alternativas Irrelevantes (IAI), propriedade em que a razão das probabilidades de escolha de duas alternativas independe dos atributos ou da disponibilidade de uma terceira alternativa.

\subsection{Elasticidade-preço no modelo de quantidade comprada}

A correta especificação da equação de demanda pelo método dos mínimos quadrados ordinários (MQO) possibilita obter a elasticidade-preço da demanda, diretamente pelo coeficiente da variável "preço da marca" numa equação de regressão com a variável dependente "quantidade vendida", desde que ambas as variáveis sejam transformadas em logaritmo natural, o que também auxilia na normalização das vendas (a não normalidade das vendas pode tornar impróprios os testes de significância e intervalos de confiança, como observa Walters \& Bommer, 1996).

Supondo uma função de apenas uma variável exógena, $y=f(x)$, pode-se diferenciar $\ln y$ em relação a $\ln x$, ou seja, $d \ln y / d \ln x$. Definindo-se $v=\ln x$, tem-se $e^{v}=x=e^{\ln x}$. De acordo com Chiang (1984), definindo-se $u=\ln y$, tem-se:

$$
\eta=\frac{d \ln y}{d \ln x}=\frac{d u}{d v}=\frac{d u}{d y} \frac{d y}{d x} \frac{d x}{d v}=\left(\frac{d \ln y}{d y}\right)\left(\frac{d y}{d x}\right)\left(\frac{d e^{v}}{d v}\right)=\frac{1}{y} \frac{d y}{d x} e^{v}=\frac{1}{y} \frac{d y}{d x} x=\frac{d y}{d x} \frac{x}{y}
$$

Se $y$ representar as vendas da marca $p$ e $x$ representar seu preço, $\eta$ é a elasticidade-preço $\left(E t_{p p}\right)$ de $p$, que neste artigo é decomposta em elasticidade-preço da quantidade comprada $\left(E q_{p p}\right)$ e elasticidade-preço da escolha da marca $\left(E e_{p p}\right)$. Se $y$ representar as vendas da marca $p$ e $x$ representar o preço da marca $j, \eta$ é a elasticidade-preço cruzada da demanda para a marca $p\left(E q_{p j}\right)$.

\subsection{Decomposição da elasticidade-preço total}

A elasticidade-preço da quantidade ( $E q_{p p}$ ) é calculada para cada uma das marcas analisadas, com base somente nas observações em que cada marca foi escolhida numa situação de compra específica. Isto é, com base no pressuposto de que "dado que o domicílio $i$ escolha a marca $p$ na ocasião $t$, ele compra a quantidade $q$ desta marca". Isto é, a quantidade comprada da marca $p$ é condicionada à sua escolha. A elasticidade-preço total da marca $p\left(E t_{p p}\right)$ é derivada por Krishnamurthi \& Raj (1988, p.11) e é dada por:

$$
E t_{p p}=E e_{p p}+E q_{p p}
$$

Portanto, de acordo com o método aqui usado, a elasticidade-preço total da marca $p$ será representada pela soma das elasticidades-preço da escolha da marca e da quantidade comprada. 


\section{Coleta dos Dados e Variáveis dos Modelos}

Os dados utilizados correspondem a um painel desbalanceado, isto é, com números diferentes de situações de compra para cada domicílio. Esta característica deve-se à exclusão do banco de dados daquelas ocasiões em que não se observou compra, pelo domicílio, nas marcas estudadas dentro da categoria de produto analisada. Foi aqui usada uma combinação das ocasiões de compra dos diversos domicílios, como num corte transversal, em que cada ocasião de compra representou uma observação independente.

Os dados provêm de domicílios que possuem cartão de fidelidade numa rede de supermercados do estado do Rio de Janeiro e referem-se a um período de 35 semanas, de julho de 2000 a março de 2001, sendo ele aqui chamado de "período de calibração do modelo". É um período razoável para este tipo de análise se comparado aos trabalhos que usaram dados escaneados em 32 semanas, como os de Tellis (1988), Guadagni \& Little (1983) e Krishnamurthi \& Raj (1991). Um viés do não controle das compras feitas pelo domicílio em locais fora da rede de supermercados analisada foi minimizado considerando-se apenas os domicílios com alta intensidade de compra na rede, pela classificação da própria empresa.

Da amostra inicial foram eliminados os domicílios com dez ou menos ocasiões de compra nas quatro marcas (que englobam $82 \%$ de participação de mercado na rede supermercadista estudada) da categoria achocolatado em pó no período de calibração. Trabalhou-se apenas com 200 domicílios obtidos aleatoriamente da amostra anterior (323 domicílios) por razões de facilidade computacional. Os 123 domicílios restantes foram usados para a averiguação da capacidade de previsão do modelo, descrita adiante. Os 200 domicílios totalizaram 2.604 ocasiões de compra, com uma média de 13,02 observações por domicílio. O intervalo médio de compra na categoria no período foi de 18 dias (2,57 semanas).

As quatro marcas foram nomeadas por A, B, C e D. As participações de mercado de cada uma eram de $64 \%$ (A), $9 \%$ (B), $5 \%$ (C) e $4 \%$ (D). Foi escolhido o tamanho mais usual na categoria, o equivalente à embalagem de $500 \mathrm{~g}$. Apenas a marca B apresenta embalagem de $400 \mathrm{~g}$.

As variáveis utilizadas foram:

Compra anterior ( COMPRA $_{p}$ ) - É um vetor de variáveis categóricas para cada marca. $\mathrm{COMPRA}_{p}=1$ se a marca $p$ foi escolhida na última compra da categoria; ou 0 , caso contrário. Esta variável foi sugerida por Neslin (2002) para modelar o estado de dependência do domicílio. Como se trata de uma variável defasada, a primeira observação de cada domicílio foi perdida, totalizando-se a amostra de 2.404 ocasiões de compra.

ESCOLHA - É a variável dependente no modelo logit $(\mathrm{ESCOLHA}=1$ se a marca foi a escolhida na ocasião $t ; 0$ no caso contrário).

Intensidade de Consumo (INTENS) - Para cada domicílio e em cada ocasião de compra calculou-se a média (em gramas) de compras na categoria. Classificou-se, então, cada domicílio em um grupo de consumo intenso (INTENS $=1$ ) ou pouco intenso (INTENS $=0$ ), se a média de compra de cada domicílio no período fosse maior (ou igual) ou menor, respectivamente, que a média de todos os domicílios (que foi de 780 gramas por ocasião de compra no período de 35 semanas). Esta é, portanto, uma variável específica de cada domicílio e invariável às marcas e ao tempo, captando parte da heterogeneidade do domicílio.

Preço por 100 gramas $\left(\right.$ PREÇO $\left._{p}\right)$ - Este vetor de variáveis foi usado no modelo de escolha e o seu logaritmo natural no modelo de quantidade comprada. Foi calculado dividindo-se o 
preço da embalagem da marca comprada pelo peso da embalagem em gramas e multiplicando-se o resultado por 100 . Desta maneira trabalhou-se com uma variável contínua (preço por 100g).

Quantidade em 100 gramas $\left(Q U A N T_{p}\right)$ - O logaritmo desta variável é a variável dependente nas equações de demanda. Foi calculada multiplicando-se o número de embalagens compradas pelo peso da embalagem em gramas, dividindo-se o resultado por 100.

$\boldsymbol{R E N D A}$ - Refere-se à renda per capita mensal do domicílio em Reais, dividida por 1.000.

Tamanho da família (TAM_FAM) - é uma variável que capta parte da heterogeneidade do domicílio e sua inclusão é recomendada por Krishnamurthi \& Raj (1991). Supõe-se que seja importante na explicação da quantidade comprada.

As variáveis demográficas $R E N D A$ e $T A M_{-} F A M$ foram obtidas pelas informações do cartão de fidelidade de cada domicílio disponíveis na rede varejista analisada.

O software estatístico SAS (versão 8) foi usado na fase inicial do tratamento dos dados e na construção das variáveis para posterior análise econométrica pelo software LIMDEP (versão 7.0).

\section{Resultados}

Os resultados dos modelos de escolha da marca de quantidade comprada são descritos a seguir. São também discutidas a validação dos modelos e a decomposição da elasticidade. A Tabela 1 traz as estatísticas descritivas, com valores de média, desvio padrão e número de observações das variáveis dos modelos de escolha da marca e quantidade comprada.

Tabela 1 - Estatísticas descritivas das variáveis dos modelos.

\begin{tabular}{l|c|cc}
\hline \multicolumn{1}{c|}{ Variável } & Média & $\begin{array}{c}\text { Desvio } \\
\text { Padrão }\end{array}$ & $\begin{array}{c}\text { Número } \\
\text { Observações }\end{array}$ \\
\hline COMPRA $_{A}$ & $0,747^{*}$ & & 2.404 \\
COMPRA $_{B}$ & $0,136^{*}$ & & 2.404 \\
COMPRA $_{C}$ & $0,131^{*}$ & & 2.404 \\
COMPRA $_{D}$ & $0,026^{*}$ & & 2.404 \\
INTENS $_{\text {PREÇO }_{A}}$ & 0,361 & & 2.604 \\
PREÇO $_{B}$ & 0,434 & 0,051 & 2.604 \\
PREÇO $_{C}$ & 0,567 & 0,026 & 2.604 \\
PREÇO $_{D}$ & 0,224 & 0,025 & 2.604 \\
QUANT $_{A}$ & 0,327 & 0,024 & 2.604 \\
QUANT $_{B}$ & 7,677 & 5,048 & 1849 \\
QUANT $_{C}$ & 6,063 & 3,847 & 347 \\
QUANT $_{D}$ & 9,985 & 7,639 & 339 \\
RENDA $_{\text {TAM_FAM }}^{7,319}$ & 3,794 & 69 \\
\hline
\end{tabular}

*por serem variáveis categóricas, todas as informações estão contidas na média. 


\subsection{Modelo de escolha da marca}

O Gráfico 1 descreve a flutuação da variável $\operatorname{PREÇO}_{P}$ ao longo do período analisado de 35 semanas. Os valores são a média dos preços pagos por todos os domicílios em cada semana. A marca B é a de maior preço, seguida pelas marcas A, D e C.

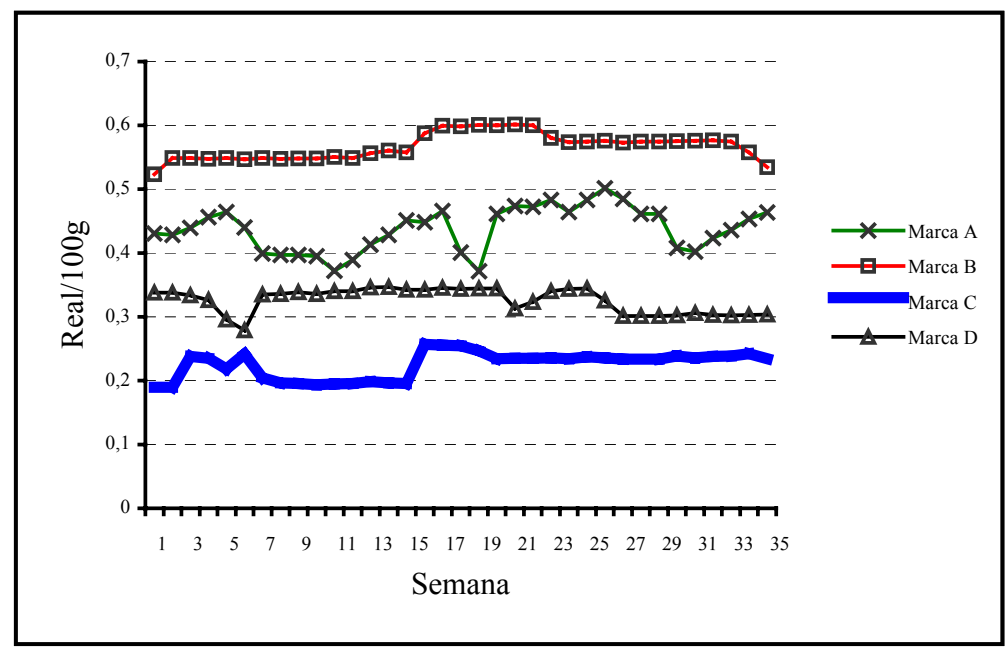

Gráfico 1 - Flutuação dos preços das quatro marcas nas 35 semanas analisadas.

Quatro funções de utilidade foram especificadas, de acordo com a equação genérica abaixo:

$$
\mathrm{U}_{P i t}=\gamma_{P 1}+\gamma_{P 2} \text { PREÇO }_{P t}+\gamma_{P 3} \text { RENDA }_{t}+\gamma_{P 4} \text { COMPRA }_{P}
$$

Os coeficientes destas funções de utilidade estão descritos na Tabela 2. Outras especificações foram construídas, utilizando-se variáveis categóricas como presença de propaganda da marca feita pela rede varejista e presença de promoção por encartes em jornais e folhetos nas lojas. Tais variáveis não foram significativas pelo teste $t$. O valor do logaritmo da função de verossimilhança de $-907,6$ apresentado na Tabela 2 , foi o maior valor entre todas as especificações testadas, indicando que esta é a mais adequada especificação.

Aplicou-se o teste de McFadden (McFadden, 1987) para testar a propriedade de IAI do modelo logit. Optou-se por testar a correlação dos componentes de erro dos modelos do subconjunto das marcas A e B e do subconjunto das marcas C e D. O argumento para o primeiro subconjunto é que as marcas A e B, nesta ordem, são as mais conhecidas perante o público, pois são mais tradicionais no mercado e contam com maiores investimentos promocionais. $\mathrm{O}$ argumento para o segundo subconjunto é que as marcas $\mathrm{C}$ e D são as de menor preço dentre as quatro marcas analisadas e podem estar posicionadas na mente dos clientes como um grupo de marcas de menor preço dentro da categoria achocolatados. Pelo teste, os componentes de erro dos modelos das marcas A e B nas funções de utilidade não são correlacionados, assim como os das marcas C e D. A propriedade IAI aplica-se ao modelo original e, por conseguinte, a estrutura simples do modelo (e não a hierárquica) está correta. 
Para a avaliação da adequação geral os modelos probabilísticos trazem dificuldades especiais. Enquanto que modelos de regressão linear apresentam os resíduos e o Coeficiente Múltiplo de Determinação $\left(R^{2}\right)$ como indicadores diretos de adequação, um modelo logit prevê apenas probabilidades que devem, então, ser comparadas com as escolhas reais. A medida $U^{2}$ apresentada por Guadagni \& Little (1983) pode ser descrita como a fração de incerteza (entropia), ou o grau de variabilidade, empiricamente explicada pelo modelo relativo a uma distribuição previamente estabelecida de probabilidades de escolha. A distribuição prévia constitui o modelo nulo, que define a máxima entropia para a situação em questão. $U^{2}$ é dado por:

$$
U^{2}=1-\frac{\ln L_{\Omega}}{\ln L_{\omega}}, \quad 0 \leq U^{2}<1
$$

onde $L_{\Omega}$ refere-se ao máximo da função de verossimilhança quando maximizada com relação a todos os parâmetros $\beta$ (incluindo o termo constante), e $L_{\omega}$ é o máximo da função de verossimilhança quando maximizada em relação apenas ao termo constante (modelo nulo) (Maddala, 1983).

Tabela 2 - Coeficientes do modelo de escolha da marca.

\begin{tabular}{ccccc}
\hline Variável & \multicolumn{2}{c|}{ Coeficiente } & estatística-t \\
\hline Constante-A & $\gamma_{A 1}$ & $-1,009$ & $(-0,508)$ \\
Constante-B & $\gamma_{B 1}$ & $-2,892$ & $(-0,928)$ \\
Constante-C & $\gamma_{C 1}$ & $-1,956$ & $(-0,906)$ \\
Constante-D & $\gamma_{D 1}$ & zero para identificação \\
PREÇO $_{A}$ & $\gamma_{A 2}$ & $-3,413^{* *}$ & $(-2,663)$ \\
PREÇO $_{B}$ & $\gamma_{B 2}$ & $-6,243^{* *}$ & $(-5,956)$ \\
PREÇO $_{C}$ & $\gamma_{C 2}$ & $-13,961^{* *}$ & $(-5,213)$ \\
PREÇO $_{D}$ & $\gamma_{D 2}$ & $-10,401 * *$ & $(-5,456)$ \\
RENDA & $\gamma_{A 3}$ & zero para identificação \\
RENDA $_{\text {RENDA }}$ & $\gamma_{B 3}$ & 0,084 & $(0,957)$ \\
RENDA $_{C 3}$ & $\gamma_{D 3}$ & $-0,418^{* *}$ & $(-2,728)$ \\
COMPRA $_{A}$ & $\gamma_{A 4}$ & $0,579 *$ & $(-2,700)$ \\
COMPRA $_{B}$ & $\gamma_{B 4}$ & $4,092^{* *}$ & $(0,676)$ \\
COMPRA $_{C}$ & $\gamma_{C 4}$ & $4,079 * *$ & $(17,660)$ \\
COMPRA $_{D}$ & $\gamma_{D 4}$ & $2,816^{* *}$ & $(16,031)$ \\
\hline Log Verossimilhança $_{U^{2}}$ & & $-907,6$ & $(8,149)$ \\
\hline
\end{tabular}

* significativo $95 \%$ probabilidade; $* *$ significativo $99 \%$ probabilidade. 
O valor de $U^{2}$ de 0,601 na Tabela 2 indica que $60,1 \%$ da entropia é explicada pelo modelo. Um modelo que prevê corretamente $100 \%$ das observações apresenta um $U^{2}=1$, ou seja, prevê corretas probabilidades 0 e 1 . A seguir são apresentadas a interpretação e a discussão dos coeficientes das variáveis do modelo de escolha e as elasticidades-preço:

Constantes específicas às alternativas - tais constantes seriam zero se todas as variáveis que explicassem as diferenças entre as marcas fossem incluídas num modelo logit. $\mathrm{Na}$ Tabela 2 estas constantes não mostraram diferença significativa de zero. Por motivo de identificação, um dos coeficientes (o da marca D) foi igualado a zero.

PREÇO $_{P}$ - Como em Krishnamurthi \& Raj (1988), a especificação correta das funções de utilidade permite a estimação dos quatro coeficientes para $\mathrm{PREÇO}_{p}$. Os parâmetros de $\mathrm{PREÇO}_{p}$ são todos negativos, como esperado, e significativos com pelo menos $95 \%$ de probabilidade. Isto indica que quanto maior o preço da marca, menos provável é sua escolha pelo domicílio. Logo, um aumento em Real/grama apresenta desutilidade marginal para as quatro marcas e os domicílios preferem um preço menor quando optam pelas marcas A, B, C e D. Contudo, o efeito da variação relativa do preço possui uma interpretação mais importante para o marketing, que é medido pela elasticidade-preço de escolha da marca, calculada pela equação 4 e mostrada na Tabela 3 para as quatro marcas. Os valores em negrito referem-se às $E e_{p p}$ e os demais, às $E e_{p j}$.

Tabela 3 - Elasticidades-preço da escolha da marca.

\begin{tabular}{c|c|c|c|c}
\hline Marcas & A & B & C & D \\
\hline $\mathbf{A}$ & $\mathbf{- 0 , 4 5 9}$ & 0,242 & 0,299 & 0,113 \\
$\mathbf{B}$ & 1,116 & $\mathbf{- 1 , 5 5 5}$ & 0,299 & 0,113 \\
$\mathbf{C}$ & 1,116 & 0,242 & $\mathbf{- 2 , 0 2 9}$ & 0,113 \\
$\mathbf{D}$ & 1,116 & 0,242 & 0,299 & $\mathbf{- 4 , 4 1 6}$ \\
\hline
\end{tabular}

Pela Tabela 3, um aumento de $1 \%$ no preço da marca A, por exemplo, causa uma redução, em média, de $0,46 \%$ na probabilidade de escolha desta marca, ceteris paribus. Em média, o mesmo aumento de $1 \%$ no preço da marca A acarreta um aumento de $1,12 \%$ na probabilidade de escolha das demais marcas, ceteris paribus, que é exatamente $E e_{p j}$. A marca $\mathrm{A}$, aquela de maior participação de mercado, apresenta a maior elasticidade cruzada da escolha, seguida pelas marcas $\mathrm{C}, \mathrm{B}$ e $\mathrm{D}$, nesta ordem. $\mathrm{O}$ fato de a marca $\mathrm{A}$ ser a menos elástica dentre as quatro parece justificado pela sua alta participação de mercado, por ser ela mais tradicional e apresentar o maior investimento de promoção.

RENDA - Basicamente esta variável reflete as diferenças em preferências pelas marcas em função da renda per capita. Selecionou-se a marca A como base para esta variável, ou seja, o coeficiente $\gamma_{A 3}$ é zero por motivos de identificação. O que interessa é o efeito da renda sobre a utilidade relativa de B, C e D. Como A é a segunda marca mais cara, após a marca B, espera-se que as marcas $\mathrm{C}$ e $\mathrm{D}$ recebam contribuições negativas de utilidade da variável RENDA em relação à marca $\mathrm{A}$, ao contrário da marca $\mathrm{B}$, pelo fato desta ser a marca mais cara. É exatamente isto que o sinal positivo de $\gamma_{B 3}$ e os sinais negativos de $\gamma_{C 3}$ e $\gamma_{D 3}$ mostram. 
COMPRA $_{P}-\mathrm{O}$ coeficiente desta variável mede o estado de dependência para com a marca. Um coeficiente positivo e significativo indica que os hábitos de escolha dos domicílios na categoria analisada são difíceis de mudar. Neste artigo, todos os coeficientes são significativos, exceto o da marca A. Portanto, a opção pelas marcas B, C ou D numa ocasião de compra influencia positivamente a probabilidade de escolha das mesmas marcas numa ocasião posterior, ou seja, há presença de estado de dependência para estas marcas.

O efeito marginal de COMPRA na probabilidade de escolha da marca D, por exemplo, é representado no Gráfico 2 pela diferença (observada no eixo da ordenada) entre as duas funções no ponto em que $\mathrm{PREÇO}_{D}=\mathrm{R} \$ 0,33$, ou seja, na média amostral de $\mathrm{PREÇO}_{D}$. O valor deste efeito no gráfico é de aproximadamente 0,062 (ou 6,2\%), mostrando que a probabilidade de um domicílio $i$ escolher a marca $\mathrm{D}$ na próxima compra será $6,2 \%$ maior se tal domicílio tiver optado por $\mathrm{D}$ na última compra.

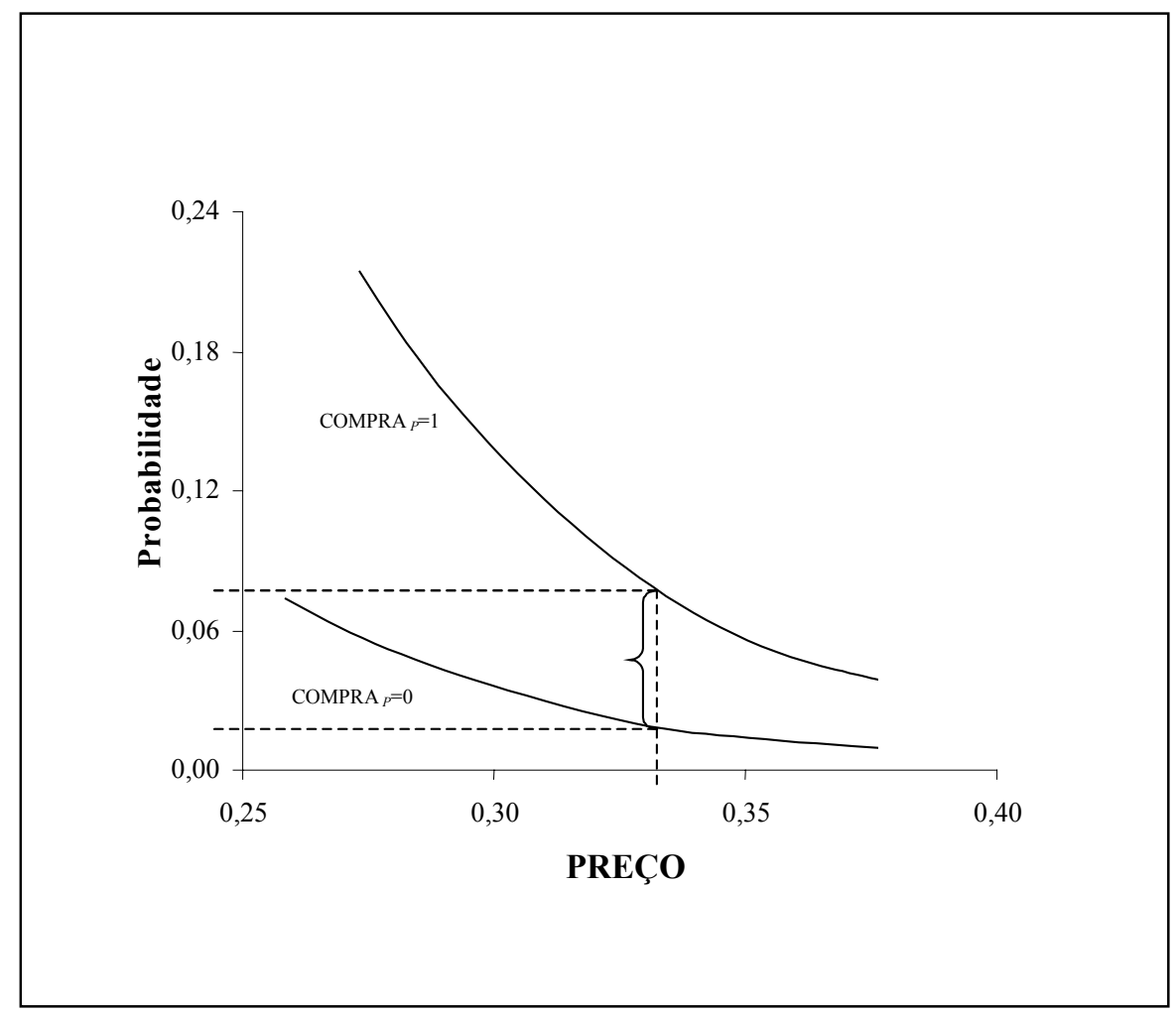

Gráfico 2 - Efeito marginal da variável COMPRA ${ }_{D}$ na probabilidade de escolha de D.

A validação do modelo logit foi feita por probabilidades previstas, usando-se a mesma amostra, e por sua capacidade de previsão na segunda amostra (123 domicílios). No primeiro caso, as probabilidades previstas para cada ocasião de compra são obtidas pela substituição na equação 2 dos valores das observações das variáveis utilizadas e dos coeficientes estimados. Com as médias amostrais para cada alternativa (Tabela 1) e os coeficientes $\gamma$ (Tabela 2) pode-se calcular as probabilidades previstas para cada marca. Primeiro calculam-se os expoentes da 
equação 2, por exemplo: $\mathrm{A}=\gamma_{A 1}+\gamma_{A 2} \times 0,434+\gamma_{A 3} \times 1,144+\gamma_{A 4} \times 0,747 . \mathrm{O}$ próximo passo é substituir os valores acima na mesma equação: $\operatorname{Prob}(\mathrm{Y}=\mathrm{A})=\frac{e^{A}}{e^{A}+e^{B}+e^{C}+e^{D}}=0,824$.

Substituindo-se os valores de $e^{B}, e^{C}$ e $e^{D}$ no numerador da equação 2, tem-se também as probabilidades médias previstas para as marcas B, C e D, que são de 0,020, 0,042 e 0,114, respectivamente. Vê-se, então, que a marca $\mathrm{A}$, em média, é a mais popular dentre as quatro alternativas.

A vantagem de trabalhar-se com probabilidades previstas é a possibilidade de simulações e avaliação de ações promocionais alternativas. Podem-se mudar os valores das variáveis, como o preço, para simular as probabilidades resultantes em tais situações e, com isto, construir cenários diferentes. Tal prática pode ser de grande implicação gerencial, visto que promoções de preço são muitas vezes feitas no varejo sem estudos de seus impactos (Doyle, 1986).

A Tabela 4 expõe os valores reais e previstos de probabilidade do modelo de escolha. Das 2.404 observações, este modelo previu corretamente as escolhas de 1.986, isto é, 82,6\%. Entretanto, a capacidade de previsão do modelo varia de acordo com a alternativa. Um excelente índice é obtido para a marca A: 88,8\%. Bons índices também são obtidos para as marcas B e C: $71,0 \%$ e 72,6\%, respectivamente. Mas o modelo previu corretamente apenas $23,0 \%$ das escolhas para a marca $\mathrm{D}$, que pode ser devido ao baixo número de observações para esta marca (69).

Tabela 4 - Capacidade de previsão do modelo de escolha da marca.

\begin{tabular}{|c|c|c|c|c|c|}
\hline \multirow[t]{2}{*}{ Previsto } & \multicolumn{4}{|c|}{ Marcas } & \multirow[t]{2}{*}{ Total } \\
\hline & $\mathbf{A}$ & B & $\mathbf{C}$ & D & \\
\hline A & 1.514 & 79 & 74 & 38 & 1.705 \\
\hline B & 83 & 230 & 7 & 4 & 324 \\
\hline $\mathrm{C}$ & 73 & 8 & 228 & 5 & 314 \\
\hline $\mathrm{D}$ & 35 & 7 & 5 & 14 & 61 \\
\hline Total & 1.705 & 324 & 314 & 61 & 2.404 \\
\hline Capacidade de Previsão & $88,8 \%$ & $71,0 \%$ & $72,6 \%$ & $23,0 \%$ & $82,6 \%$ \\
\hline
\end{tabular}

Noutra validação do modelo usou-se a segunda amostra (123 domicílios em 1.491 ocasiões de compra). A principal pergunta nesta validação é: o quão bem o modelo prevê a participação de mercado das marcas em uma amostra obtida no mesmo período de análise, mas composta por domicílios diferentes daqueles usados na sua calibração? A resposta a esta pergunta está no Gráfico 3, com a média das participações de mercado real e prevista da marca $p\left(m_{p}\right)$ para cada período de cinco semanas. Tal medida é representada pela linha pontilhada. As linhas contínuas que formam o intervalo de confiança de $90 \%$ foram obtidas pelos valores de $\pm 1,64$ desvio padrão de $m_{p}$. 


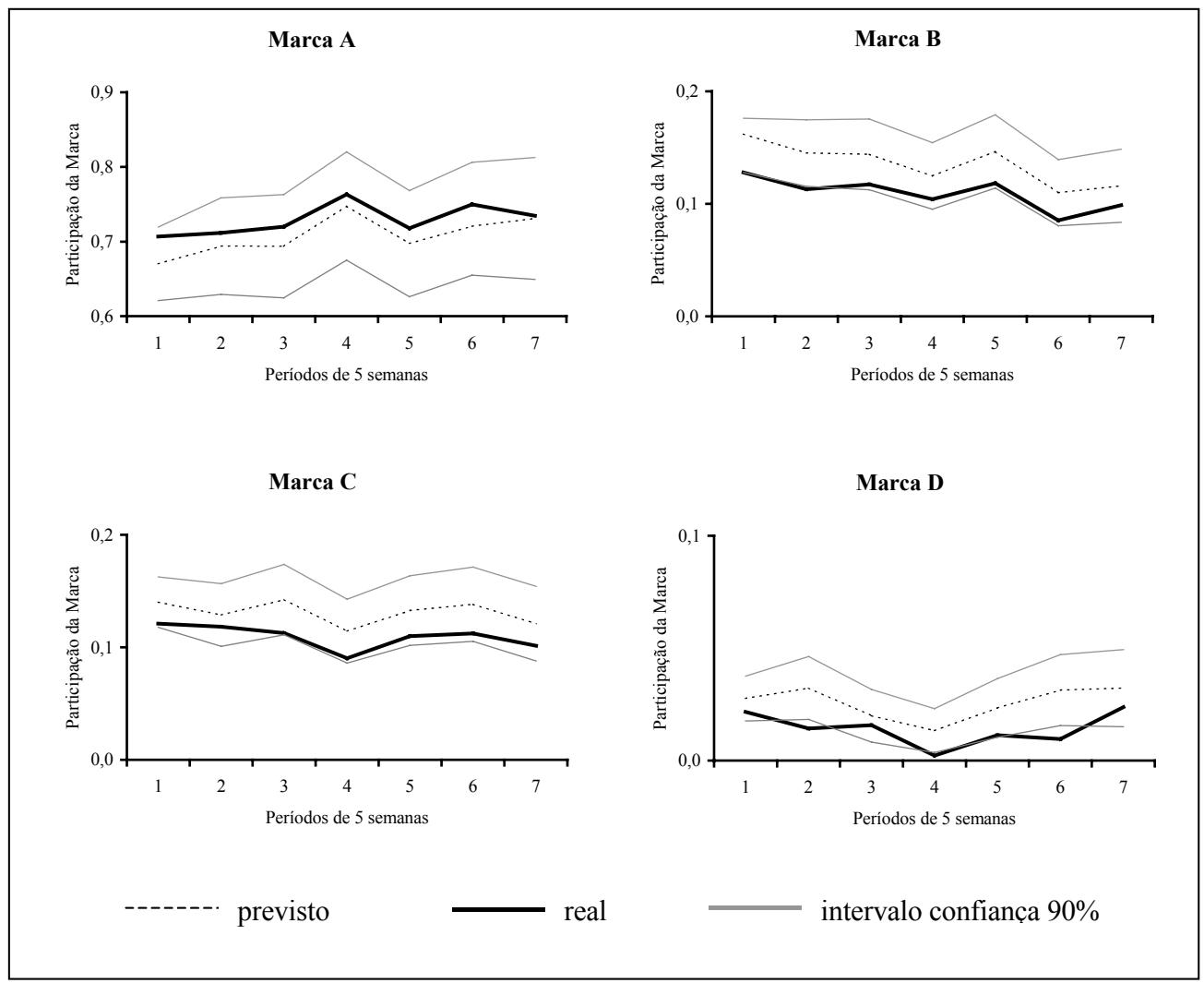

Gráfico 3 - Flutuações das participações de mercado real e prevista para as quatro marcas (amostra de validação).

Pelo Gráfico 3, os valores previstos de participação de mercado estão dentro do intervalo de confiança de $90 \%$ praticamente durante todo o período de análise para as quatro marcas (com exceção da marca D, corroborando a baixa capacidade de previsão para esta marca evidenciada na Tabela 4). O modelo apresenta boa capacidade de captar os picos e a tendência da participação de mercado das marcas A, B e C ao longo do tempo.

\subsection{Modelo da quantidade comprada}

As quatro equações de demanda foram baseadas em amostras de 1.849, 347, 339 e 69 observações, respectivamente para as marcas A, B, C e D. Os coeficientes e outras estatísticas deste modelo estão na Tabela 5. A multicolinearidade e a heteroscedasticidade foram examinadas antes da especificação do modelo final de análise. A primeira não foi um problema para a especificação pela análise dos Fatores de Inflação de Variância (Hair et al., 1998). Já a heteroscedasticidade foi detectada pelo teste de Breusch-Pagan (Gujarati, 2000). Assim, os desvios padrão dos coeficientes destas regressões foram corrigidos pelo método de White (White, 1980). A seguir, os coeficientes de cada variável e seus testes estatísticos são interpretados: 
$\ln$ PREÇO $_{p}$ - Na Tabela 5 os coeficientes $\alpha_{j p}$ representam as elasticidades-preço. Quando $j=p$, tais coeficientes exprimem as $E q_{p p}$, cujos valores e respectivas estatísticas de $t$ estão sombreadas na Tabela 5. Quando $j \neq p$, estes coeficientes correspondem às $E q_{p j}$. Os coeficientes devem ser lidos na tabela de acordo com a ordem de seus subscritos. Por exemplo, sendo $\alpha_{B A}=0,488$, caso o coeficiente fosse significativo, um aumento do preço da marca $\mathrm{B}$ em $1 \%$ provocaria um aumento da quantidade demandada da marca $\mathrm{A}$ em aproximadamente $0,5 \%$.

Tabela 5 - Coeficientes das equações de demanda.

\begin{tabular}{|c|c|c|c|c|c|}
\hline \multirow[b]{2}{*}{ Variável } & \multirow[b]{2}{*}{ Coef. } & \multicolumn{4}{|c|}{ Marcas } \\
\hline & & A & B & C & D \\
\hline \multirow[t]{2}{*}{ Intercepto } & $\alpha_{p 0}$ & $1,235^{*}$ & $2,612 * *$ & $2,618^{*}$ & $1,625^{*}$ \\
\hline & & $(2,754$ & $(3,126)$ & $(2,490)$ & $(2,521)$ \\
\hline \multirow[t]{2}{*}{$\operatorname{lnPREÇO}{ }_{A}$} & $\alpha_{A p}$ & $-0,159 *$ & 0,082 & 0,053 & 0,096 \\
\hline & & $(-2,078)$ & $(0,418)$ & $(0,196)$ & $(0,312)$ \\
\hline \multirow[t]{2}{*}{$\operatorname{lnPREÇO}{ }_{B}$} & $\alpha_{B p}$ & 0,488 & $-0,025$ & $-0,037$ & 0,012 \\
\hline & & $(1,808)$ & $(-0,338)$ & $(-0,052)$ & $(0,020)$ \\
\hline \multirow[t]{2}{*}{$\operatorname{lnPREÇO}{ }_{C}$} & $\alpha_{C p}$ & 0,028 & 0,169 & $-0,349 *$ & 0,157 \\
\hline & & $(0,260)$ & $(0,632)$ & $(-1,963)$ & $(0,437)$ \\
\hline \multirow[t]{2}{*}{$\operatorname{lnPREÇO_{D}}$} & $\alpha_{D p}$ & $-0,103$ & 0,171 & 0,155 & $-0,081$ \\
\hline & & $(-0,799)$ & $(0,549)$ & $(0,407)$ & $(-1,545)$ \\
\hline \multirow[t]{2}{*}{ INTENS } & $\alpha_{p 6}$ & $0,511 * *$ & $0,579 * *$ & $0,463 * *$ & $0,289 *$ \\
\hline & & $(22,187)$ & $(11,218)$ & $(8,845)$ & $(2,167)$ \\
\hline \multirow[t]{2}{*}{ TAM_FAM } & $\alpha_{p 7}$ & 0,011 & 0,011 & 0,087 & 0,057 \\
\hline & & $(1,672)$ & $(1,651)$ & $(0,541)$ & $(0,772)$ \\
\hline$R^{2}$ ajustado & & 0,28 & 0,39 & 0,31 & 0,53 \\
\hline $\mathbf{N}$ & & 1.849 & 347 & 339 & 69 \\
\hline
\end{tabular}

Estatística t entre parênteses: * significativo $95 \%$ de probabilidade; ** significativo $99 \%$ de probabilidade.

Nem todos os sinais de $\alpha_{j p}(j \neq p)$ são positivos, como esperado. Mas a nenhuma conclusão se pode chegar sobre os sinais dos coeficientes, visto que eles não são significativamente diferentes de zero. Logo, a variação dos preços das marcas não preferidas pelo domicílio não influencia a decisão da quantidade comprada da marca preferida, ou seja, as marcas apresentam baixa elasticidade-cruzada da demanda. Já todos os coeficientes $\alpha_{j p}(j=p)$ tiveram sinais negativos, como esperado, indicando que um aumento de preço na marca causa uma redução de sua quantidade vendida. Entretanto, somente os coeficientes das marcas $\mathrm{A}$ e $\mathrm{C}$ foram significativos.

TAM_FAM - O coeficiente da variável TAM_FAM foi positivo para todas as marcas, indicando que quanto maior o tamanho da família, maior a quantidade comprada das marcas.

INTENS - Os coeficientes de INTENS são significativos em todas as regressões. A categoria base nesta variável é o grupo de domicílios com baixa intensidade de consumo. Por exemplo, para a marca A, ceteris paribus, os domicílios de alta intensidade de consumo 
mostram um consumo marginal da marca de 0,51 unidades. Lembrando-se que a unidade da variável dependente é o logaritmo natural da quantidade em 100 gramas, o consumo marginal dos domicílios de consumo intenso sobre a categoria base é, então, de 166,7 gramas.

A validação das equações de demanda foi prejudicada pelos baixos valores do $R^{2}$ para todas as quatro regressões. Como mostra a Tabela 4 , apenas 28 a 53\% da variação na variável dependente é explicada pela variação nas variáveis independentes. Assim, optou-se por não validar o modelo para testar sua capacidade de previsão numa segunda amostra, já que quanto menor o valor de $R^{2}$, menor a confiança na capacidade de previsão da reta de regressão.

De acordo com a equação $6 \mathrm{e}$ as Tabelas 3 e 5 , as elasticidades totais $\left(E t_{p p}\right)$ para as marcas A, B, C e D, mostradas na Tabela 6, são $-0,618,-1,580,-2,378$ e $-4,497$, respectivamente. A marca A, de maior participação, é inelástica perante as demais, que são similarmente elásticas. A marca $\mathrm{D}$, de menor participação de mercado, é a mais elástica com relação ao preço. A Tabela 6 também mostra que $E e_{p p}$ é maior que $E q_{p p}$ para todas as marcas analisadas (todas as análises dos valores de elasticidades neste artigo são baseadas em valores absolutos).

Tabela 6 - Decomposição da elasticidade-preço.

\begin{tabular}{c|c|c|c|c}
\hline \multirow{2}{*}{ Elasticidade } & \multicolumn{5}{|c}{ Marcas } \\
\cline { 2 - 5 } & $\mathbf{A}$ & $\mathbf{B}$ & $\mathbf{C}$ & $\mathbf{D}$ \\
\hline$E e_{p p}$ & $-0,459$ & $-1,555$ & $-2,029$ & $-4,416$ \\
$E q_{p p}$ & $-0,159$ & $-0,025$ & $-0,349$ & $-0,081$ \\
$E t_{p p}$ & $\mathbf{- 0 , 6 1 8}$ & $\mathbf{- 1 , 5 8 0}$ & $\mathbf{- 2 , 3 7 8}$ & $\mathbf{- 4 , 4 9 7}$ \\
\hline
\end{tabular}

\section{Conclusões}

Modelar as decisões de compra (escolha e quantidade) com o método descrito neste artigo proporciona uma análise útil das diferentes naturezas das elasticidades nestes dois estágios da decisão de compra. Tal análise não é possível quando se estima apenas a elasticidadepreço da demanda total e agregada. Por exemplo, as marcas B e D apresentaram baixa elasticidade-preço da quantidade ( $\left.E q_{p p}\right),-0,025$ e $-0,081$, respectivamente (Tabela 6). Mas suas elasticidades-preço da escolha $\left(E e_{p p}\right)$ possuem magnitudes maiores $(-1,555$ e $-4,416$, respectivamente). Por outro lado, a natureza da decomposição da elasticidade-preço para a marca A é diferente. Esta marca apresenta um valor relativamente mais alto da elasticidadepreço da quantidade $\left(E q_{p p}=-0,159\right)$, mas sua elasticidade-preço da escolha da marca $\left(E e_{p p}\right)$ é relativamente baixa $(-0,459)$.

Esta estimação conjunta tem implicações gerenciais úteis. Para muitas categorias de produtos, promoções de preço são mais prováveis de induzir à mudança da marca do que resultar em aumento de quantidade vendida para o mesmo domić́lio. Os resultados deste artigo mostram que as variações de preços, na categoria estudada, têm maior efeito de 
mudança das ou para as marcas B, C e D em relação à marca A. Altos valores da elasticidade-preço da escolha da marca $\left(E e_{p p}\right)$ sobre valores da elasticidade-preço da quantidade $\left(E q_{p p}\right)$, como no caso das marcas B, C e D, indicam que promoções de preço visando aumentar o consumo dos domicílios que preferem tais marcas não são decisões inteligentes, pois elas têm efeito de captar novos domicílios para as respectivas marcas e não o aumento da quantidade comprada por parte daqueles que normalmente já as compram. Já a marca A apresenta um maior equilíbrio entre as magnitudes das duas elasticidades, embora a elasticidade-preço da escolha da marca $\left(E e_{p p}\right)$ ainda seja maior que a elasticidade-preço da quantidade $\left(E q_{p p}\right)$. Logo, não só a elasticidade-preço apresenta alta variação entre marcas na mesma categoria, mas suas magnitudes decompostas também, reclamando diferentes decisões e ações de preço para diferentes marcas e objetivos de promoção de preços. A decomposição da elasticidade-preço pode, portanto, otimizar as promoções de preço no varejo por fornecer informações mais específicas sobre marcas específicas.

Além do preço, outras variáveis foram usadas nos modelos e possibilitaram informações relevantes. No modelo de escolha da marca, os coeficientes da variável $\mathrm{COMPRA}_{P}$, por exemplo, permitem inferir uma certa lealdade às marcas $\mathrm{B}, \mathrm{C}$ e $\mathrm{D}$. Os consumidores apresentam estado de dependência por estas marcas ao longo do período de análise. No modelo de quantidade comprada, os coeficientes de TAM FAM indicam que o tamanho da família, como esperado, explica a quantidade comprada, mas não explica a escolha da marca (a variável não foi significativa neste modelo).

O modelo de escolha da marca apresentou uma boa capacidade de previsão $(82,6 \%)$ de escolhas. $\mathrm{O}$ método aqui empregado permite trabalhar com probabilidades previstas, tendo a vantagem da possibilidade de simulações. Podem-se construir cenários de táticas de promoções de preços pela simulação de diferentes níveis de preços e comparação das participações de cada marca dentro do mix de marcas das empresas que vierem a utilizar este instrumento.

Uma limitação da pesquisa é que ela é baseada nas escolhas dos consumidores pela marca e quantidade comprada, mas sabe-se que as escolhas são mais complexas, pois abrangem ainda quando e onde comprar, além das unidades de manutenção de estoque, como sabores e tamanhos de embalagem, que não foram analisadas neste artigo. Pesquisas futuras com dados escaneados no Brasil podem vir a considerar tais escolhas.

\section{Agradecimentos}

O autor é grato à CAPES (Coordenação de Aperfeiçoamento de Pessoal de Nível Superior) pelo apoio financeiro à realização desta pesquisa.

\section{Referências Bibliográficas}

(1) Ben-Akiva, M. \& Lerman, S. (1985). Discrete Choice Analysis: Theory and Application to Travel Demand. MIT Press, Cambridge, Massachusetts.

(2) Chiang, A.C. (1984). Fundamental Methods of Mathematical Economics. $3^{\text {rd }}$ ed. McGraw-Hill, Singapore.

(3) Chintagunta, P.K.; Kyriazidou, E. \& Pertold, J. (2001). Panel data analysis of household brand choices. Journal of Econometrics, 103(1), 111-153. 
(4) Doyle, P. (1986). Measuring the true profitability of sales promotions. The Journal of the Operational Research Society, 37(10), 955-966.

(5) Gijsbrechts, E. (1993). Prices and pricing research in consumer marketing: some recent developments. International Journal of Research in Marketing, 10(2), 115-151.

(6) Guadagni, P.M. \& Little, J.D.C. (1983). A logit model of brand choice calibrated on scanner data. Marketing Science, 2(3), 203-238.

(7) Gujarati, D. (2000). Econometria Básica. Makron Books, São Paulo.

(8) Hair, J.F. Jr.; Anderson, R.E.; Tatham, R.L. \& Black, W.C. (1998). Multivariate Data Analysis. Prentice Hall, Inc., Upper Saddle River, New Jersey.

(9) Kim, D. (1996). Interaction of advertising exposures, lagged choice, and price sensitivity in the multidimensional discrete choices. Ph.D. Thesis, University of Iowa, Iowa City, USA.

(10) Krishnamurthi, L. \& Raj, S.P. (1988). A model of brand choice and purchase quantity price sensitivities. Marketing Science, 7(1), 1-20.

(11) Krishnamurthi, L. \& Raj, S.P. (1991). An empirical analysis on the relationship between brand loyalty and consumer price elasticity. Marketing Science, 10(2), 172-183.

(12) Liao, T.F. (1994). Interpreting probability models: logit, probit, and other generalized linear models. Sage, Thousand Oaks, 88p. (Series in quantitative applications in the social sciences, no. 07-101).

(13) Maddala, G.S. (1983). Limited Dependent and Qualitative Variables in Econometrics. Cambridge University Press, New York, NY.

(14) McFadden, D. (1987). Regression based specification tests for the multinomial logit model. Journal of Econometrics, 34(1), 63-82.

(15) Neslin, S.A. (2002). Sales Promotion. Marketing Science Institute, Cambridge, Massachusetts.

(16) Tellis, G. (1988). Advertising exposure, loyalty, and brand purchase: a two stage model of choice. Journal of Marketing Research, 25(2), 134-144.

(17) Verbeek, M. (2000). A Guide to Modern Econometrics. John Wiley \& Sons, New York, NY.

(18) Walters, R.G. \& Bommer, W. (1996). Measuring the impact of product and promotionrelated factors on product category price elasticities. Journal of Business Research, 36(3), 203-216.

(19) White, H. (1980). A heteroscedasticity-consistent covariance matrix estimator and a direct test for heteroscedasticity. Econometrica, 44(3), 817-838. 